\title{
Observing Crop Growth and Vitality with the Copernicus Mission
}

\section{Conference Paper}

\section{Author(s):}

Yurugullu, Onur; Liebisch, Frank

Publication date:

2018

Permanent link:

https://doi.org/10.3929/ethz-b-000306058

Rights / license:

In Copyright - Non-Commercial Use Permitted

Originally published in:

https://doi.org/10.1109/igarss.2018.8517298 


\title{
OBSERVING CROP GROWTH AND VITALITY WITH THE COPERNICUS MISSION
}

\author{
Onur Yuzugullu ${ }^{1}$, Frank Liebisch ${ }^{2}$ \\ ${ }^{[1]}$ AgriCircle AG, Rapperswil, Switzerland \\ ${ }^{[2]}$ Institute of Agricultural Sciences, ETH Zurich, Switzerland
}

\begin{abstract}
In this study, we show and investigate the use of optical and synthetic aperture radar (SAR) based remote sensing opportunities provided by the Copernicus satellite mission for agricultural support. We investigated the temporal patterns of two major crops, sugar beet, and winter wheat. The observations were conducted using both optical and SAR data in relation to temporal availability and potential agricultural applications. The combined use of both remote sensing methods is strongly emphasized reflecting the timely need of the information by farmers and the restrictions of the remote sensing data mainly due to weather conditions.
\end{abstract}

Index Terms - Agriculture, Polarimetric SAR, Multispectral, Copernicus Mission, Temporal Analysis

\section{INTRODUCTION}

It is a common practice to use space-borne remote sensing missions to gather information supporting agriculture. Such information can be crop or land cover classification [1]; crop development, phenology, crop performance and vitality parameters [2]. Towards this aim, the space-borne remote sensing techniques, Polarimetric Synthetic Aperture Radar (PolSAR) and Multispectral Sensing (MS), are used with the latter being the most common. Both technologies have their advantages and disadvantages. MS data are used to detect the differences in plant cover, reflecting early crop growth before canopy closure and vitality parameters such as crop chlorophyll and water content [3]. Besides, Ms are also used for crop and land use classification. However, they are prone to failure due to cloud or snow cover. PolSAR data, on the other hand, can detect changes in morphology and dielectric properties of plants and is frequently used for crop classification and can be used at day and night and at all weather conditions. However, rainy conditions and water on plants can affect the backscattered SAR signal and thus the derived information.

For agriculture monitoring, today, both methods are rarely used in combination. Such an integration would offer an increase in data acquisition security and quality due to the fact that PolSAR data can bridge clouded periods and result in better biomass and growth information acquisition during periods when the crop canopy is covered and most spectral infor- mation saturates. The MS data, on the other hand, offer better crop vitality information on plant pigment contents which allow to decipher reasons for growth limits and potentially allow derivation of crop management recommendations such as demand of nitrogen fertilizer.

Here we show the temporal development of PolSAR and MS data in two major crops, winter wheat (WW) and sugar beet (SB), throughout two seasons. Thereby, we show their potential for the combined use for agricultural information retrieval and their partly redundant nature with a field scale dispersion.

\section{METHODOLOGY}

\subsection{Crop Calendar}

For this study, we selected WW and SB, two major crops cultivated in the Swiss midlands with contrasting traits and crop calendar. WW is a monocotyle and SB a dicotyle plant, reflecting an erect and horizontal growth habit, respectively. The crop calendar represents the growth and development of the crops, as affected by environmental conditions and management practices. WW is typically sown around October in the presented region so that it can establish its first three leaves before winter. Tillering and shooting generally happen in February and March, depending mostly on the climate of the respective year. Maximum canopy cover, therefore, is usually reached latest in May. Flowering takes place around mid of June and senescence and harvest in July. SB, on the other hand, is often sown in March following the field to be fallow over winter. Its establishment and growth are slow in the beginning and maximum canopy cover is usually reached in July. Harvest takes place from the end of September in early circumstances to latest November.

\subsection{Test Area}

This research was conducted in the fields around the Plant Research Station of the Swiss Federal Institute of Technology (ETH Zurich), located in Eschikon, Switzerland.The crop calendars were obtained through frequent visual observation to determine the key dates and growth irregularities of each individual phenological cycle. 


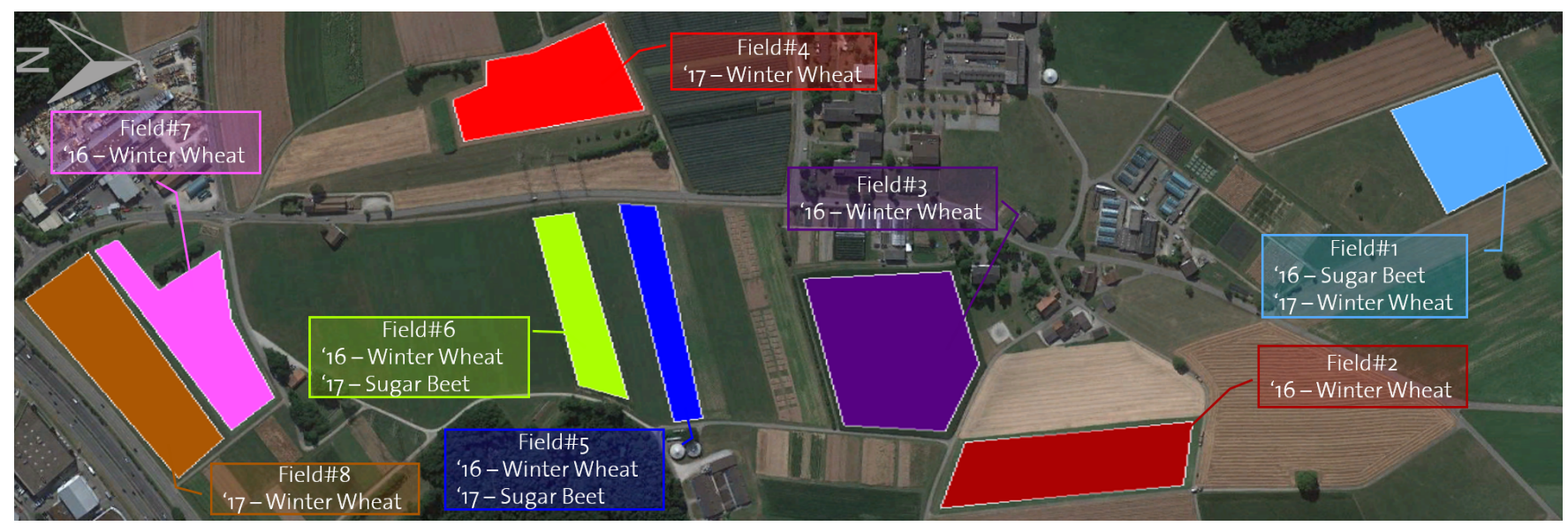

Fig. 1: The test area is located in the North-East region of Zurich. The colors in the image depict different fields and crops.

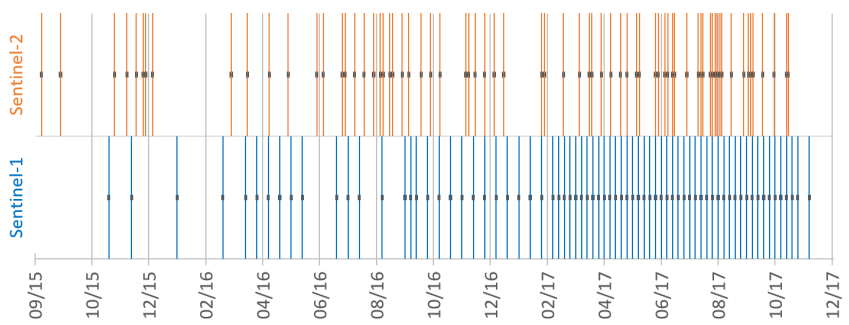

Fig. 2: Available Sentinel-1 and Sentinel-2 ( $<50 \%$ cloud cover $)$ data

\subsection{Remote sensing data from Copernicus Mission}

The Copernicus Mission is developed by the European Space Agency (ESA) under the motto of "Observing the Earth", and provide data free of charge [4]. It is a family of missions called Sentinel. Currently, each Sentinel mission is a constellation of two satellites. To access the data for this study the Google Earth Engine platform was used over its Python API. The data was downloaded for the dates between October 1, 2015, and November 30, 2017, which is presented in Fig. 2.

\subsubsection{Sentinel-1}

Sentinel-1 (S1) is a Synthetic Aperture Radar (SAR) imaging mission, which is capable of providing dual-polarized data $(\mathrm{HH}+\mathrm{HV}, \mathrm{VV}+\mathrm{VH})$ having $10 \mathrm{~m}$ spatial resolution independent of weather and light conditions. The C-band sensor has a frequency of $5.405 \mathrm{GHz}$ and wavelength of $5.5 \mathrm{~cm}$. The data was obtained in form of Ground Range Detected (GRD) scenes provided as calibrated and ortho-rectified products [5]. In this research, dual-polarization, $\mathrm{VV}+\mathrm{VH}$, backscattering intensity data with $36^{\circ}$ incidence angle was used for the monitoring purposes.

Due to the limitations of the Google Earth Engine, only the backscattering intensities in log-scale $(\mathrm{dB}), \sigma_{\mathrm{VV}}^{\mathrm{o}}$ and $\sigma_{\mathrm{VH}}^{\mathrm{o}}$, has been used for the temporal analysis. Before the analysis, the noise in the data was reduced using a $5 \times 5$ Lee-smoothing window [6]. The backscattering intensities carry information on the physical and dielectric properties of the canopies, which makes them sensitive to plant morphology.

\subsubsection{Sentinel-2}

Sentinel-2 (S2) is a multispectral optical imaging mission, which provides 13 bands in the visible and near-infrared parts of the spectrum. The bands have spatial resolutions varying in 10, 20 and 60 meters [5]. The data was provided as top of atmosphere reflectance (Level 1c). The cloud interference on the images was filtered with a 50\% threshold over the full scene before downloading. Subsequently, the scenes were filtered on pixel-level using multi-band threshold based filters [7]. Therefore, the cloud class pixels from different filters are combined to obtain a single mask. Using the cloud mask, the percentage of cloud cover over fields were calculated. The fields having more than $10 \%$ cloud cover were eliminated from the further analysis.

The first chosen multi-spectral parameter to represent the vitality of the crops through their growth cycles is the NDVI (1), Normalized Difference Vegetation Index [8]. The changes in the NDVI can be interpreted for the changes in crop cover and green vegetation biomass [3]. The second one is the MCARI2 (2), Modified Chlorophyll Absorption Ratio Index [9]. MCARI2 provides information on the chlorophyllcontaining biomass (mostly leaves). It is closely related to the photo-synthetically active leaf area of the biomass and thus a good indicator for crop development and vitality.

$$
\begin{aligned}
\mathrm{NDVI} & =(\mathrm{B} 8-\mathrm{B} 4) /(\mathrm{B} 8+\mathrm{B} 4) \\
\text { MCARI2 } & =\frac{1.5[2.5(\mathrm{~B} 8-\mathrm{B} 4)-1.3(\mathrm{~B} 8-\mathrm{B} 2)]}{\sqrt{(2 \times \mathrm{B} 8+1)^{2}-(6 \times \mathrm{B} 8-5 \sqrt{\mathrm{B} 4}-0.51)}}
\end{aligned}
$$




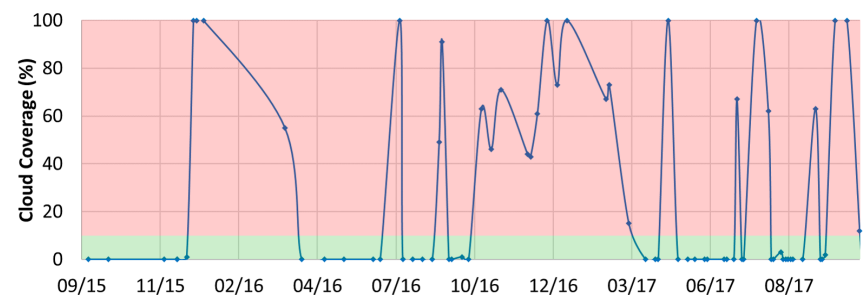

Fig. 3: Temporal trend of percent cloud cover over the Field\#5 with green shading showing used and red shading not used data.

\section{RESULTS AND DISCUSSION}

In this research, we analyzed the temporal pattern and interfield dispersion of C-band SAR and MS data from the Copernicus mission for WW and SB crops. We downloaded 74 SAR and 72 MS scenes from the test area located in Eschikon, Switzerland. For the SAR data, we were able to use the complete data set assuming negligible influence of cloud cover. For the optical data, we applied two filters. The first filter checked for 50\% cloud cover over the image, while in the second cloud cover filter we applied a pixel-based filter bundle [7] excluding the region of interests containing more than $10 \%$ pixels classified as clouds. As a result of the second cloud cover filter, 5 to $47 \%$ of the MS data was determined to be not usable. In Fig. 3, the percentage cloud cover on the Field\#5 is given. The longest phases of cloud cover were observed during winter. Considering the observed phases of cloud cover, a large portion of the data cannot be used to obtain crop information from S2. Since we use top of atmosphere reflectances, the implementation of atmospheric correction might improve the number of usable scenes slightly. Considering the fact that a large fraction of the MS data throughout the season is not usable for crop monitoring, space-borne SAR systems and below cloud sensing methods like unmanned aerial vehicles (UAVs) become important at times when space-borne MS data is not available.

In Fig. 4 we provide the temporal trends for SB (a) and WW (b) during two seasons, 2016 and 2017. Each crop is analyzed with four parameters, respectively: NDVI, MCARI, $\sigma_{\mathrm{VV}}^{\mathrm{o}}$ and $\sigma_{\mathrm{VH}}^{\mathrm{o}}$. The line colors correspond to the region of interests given in Fig. 1 for the observed fields.

In SB, Fig. 4(a), NDVI parameter shows a steady increase till August until the crop reaches maximum above-ground biomass and cover in both seasons. Later on, it reduces during the maturative stage with decreasing of leaf biomass and eventually chlorophyll content. The MCARI2, on the other hand, shows a similar pattern with a more pronounced increase towards the maximum of leaf area and biomass. This stronger increase can be explained by the fact that the MCARI2 reflects the integrated chlorophyll and the leaf area information in contrast to the biomass fraction for NDVI. For the SAR data, both $\sigma_{\mathrm{VV}}^{\mathrm{o}}$ and $\sigma_{\mathrm{VH}}^{\mathrm{o}}$ steadily increase similar to the spectral indices, but saturate earlier. The patterns show
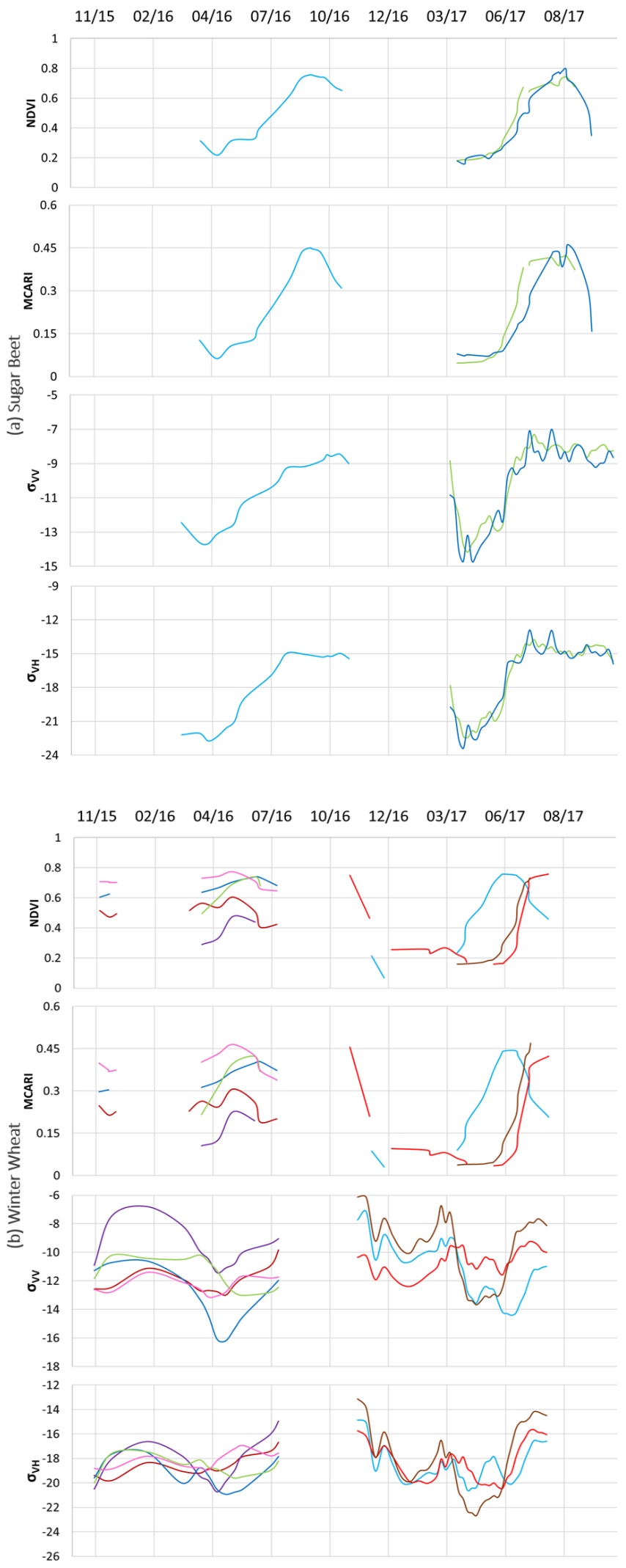

Fig. 4: Temporal trends of NDVI, MCARI, $\sigma_{\mathrm{VV}}^{o}$ and $\sigma_{\mathrm{VH}}^{o}$ for (a) SB and (b) WW fields with colors corresponding to those in Fig.1. 
similarities in both seasons, although, in 2017 higher fluctuation was observed compared to 2016. This condition might be related to the precipitation pattern differences in the two years. In 2016 the summer season was drier, while in 2017 higher precipitation frequency was observed. This affects the SAR data both by changing the morphology of plants and the dielectric properties of the leaf surfaces. Also, in 2017, the inter-field dispersion was low. The missing optical data was not critical for SB monitoring since the important phases of the growth started in spring were covered sufficiently by both sensors.

In WW fields, Fig. 4(b), the patterns show differences between seasons with high inter-field dispersion. In 2016, the large part of the optical data was missing due to high cloud cover. Thus, we are only able to comment on the growth patterns for the 2017 MS data. In WW crops, the NDVI shows a low flat line in winter and spring of the growth cycle which is followed by a sharp increase towards summer reflected by NDVI and MCARI2. During the maturative phase at the end of the growth cycle, both NDVI and MCARI2 decrease due to decreasing wet-biomass and chlorophyll content. Since SAR data is almost independent of the weather conditions, we were able to monitor the growth for both seasons. Both seasons show differences in pattern and dispersion. Considering the 2016 data, both $\sigma_{\mathrm{VV}}^{\mathrm{o}}$ and $\sigma_{\mathrm{VH}}^{\mathrm{o}}$ parameters start with an increasing trend, likely related to increasing canopy height and canopy density, followed by a decrease, caused by the end of the stem elongation, and an increase again, at the time of flag leaf formation and heading. In 2017, on the other hand, regularly occurring precipitation likely impacted the $\sigma_{\mathrm{VV}}^{\mathrm{o}}$ and $\sigma_{\mathrm{VH}}^{\mathrm{o}}$ temporal patterns. Unlike the SB fields, in WW fields the missing data significantly affected the crop monitoring by MS data. The missing optical data in early spring 2016 would have prevented a spatial fertilization support using such MS data as a basis. This could be partly compensated by the use of the available SAR data.

\section{SUMMARY AND CONCLUSIONS}

In precision agriculture using remote sensing, it is important to know the crop species to interpret the information available by the satellites correctly. This will allow deriving products such as crop growth and related fertilizer maps ready for farm applications. It is also important for the availability of MS and SAR data to provide synergistic and alternative use scenarios. This is because MS data is not always available due to cloud cover, while SAR does not inform about parameters like chlorophyll concentration. Under these terms, an effective system would combine both MS and SAR data. Even though the single use of either MS or SAR data provides meaningful information on crop growth. Only both combined ensure sufficient temporal cover and extended information on plant phenology and morphology less dependent on weather.

The above-presented study shows the need and the poten- tial of a combined use of MS and SAR based sensing methods for satellite missions aiming to support precision agriculture. Whereas a lack of data might be tolerable for monitoring purposes it is rarely for applications in crop management. The restricted time windows to conduct certain crop management measures such as spring fertilization and the nearly unpredictable cloud cover emphasizes the consideration of backup and support methodology such as implemented in the Copernicus mission to successfully derive agricultural support information in time. To facilitate the combined use of the SAR and the MS for precision agriculture, larger areas with higher number of crop types need to be studied.

\section{ACKNOWLEDGEMENT}

We would like to thank the farmers and the cantonal agricultural competence center Strickhof to whom the fields investigated, and Peter Frölich for their support during this study.

\section{REFERENCES}

[1] C. Gómez, J. C. White, and M. A. Wulder, "Optical remotely sensed time series data for land cover classification: A review," ISPRS Journal of Photogrammetry and Remote Sensing, vol. 116, pp. 55-72, 2016.

[2] O. Yuzugullu, S. Marelli, E. Erten, B. Sudret, and I. Hajnsek, "Determining rice growth stage with X-band SAR: A metamodel based inversion," Remote Sensing, vol. 9, no. 5, p. 460, 2017.

[3] F. Liebisch, G. Küng, A. Damm, and A. Walter, "Characterization of crop vitality and resource use efficiency by means of combining imaging spectroscopy based plant traits," in 6th Workshop on Hyperspectral Image and Signal Processing: Evolution in Remote Sensing (WHISPERS). IEEE, 2014, pp. 1-4.

[4] ESA. (2017) Observing the Earth. [Online]. Available: http: //www.esa.int/Our_Activities/Observing_the_Earth/Copernicus

[5] Google. (2017) Google Earth Engine Explorer. [Online]. Available: https://explorer.earthengine.google.com/\#search/tag: sentinel

[6] J.-S. Lee, "Speckle analysis and smoothing of synthetic aperture radar images," Computer graphics and image processing, vol. 17, no. 1, pp. 24-32, 1981.

[7] A. Hollstein, K. Segl, L. Guanter, M. Brell, and M. Enesco, "Ready-to-use methods for the detection of clouds, cirrus, snow, shadow, water and clear sky pixels in Sentinel-2 MSI images," Remote Sensing, vol. 8, no. 8, p. 666, 2016.

[8] J. Rouse, J. Schell, and D. Deering, "Monitoring vegetation systems in the Great Plains with ERTS," in The 3rd Earth Resources Technology Satellite-1 Symposium. Greenbelt, 1974, pp. 30917.

[9] D. Haboudane, J. R. Miller, E. Pattey, P. J. Zarco-Tejada, and I. B. Strachan, "Hyperspectral vegetation indices and novel algorithms for predicting green LAI of crop canopies: Modeling and validation in the context of precision agriculture," Remote sensing of environment, vol. 90, no. 3, pp. 337-352, 2004. 